\title{
BMJ Open Change in fasting plasma glucose and incident type 2 diabetes mellitus: results from a prospective cohort study
}

\author{
Amirhossein Mozaffary, ${ }^{1}$ Samaneh Asgari, ${ }^{1}$ Maryam Tohidi, ${ }^{1}$ \\ Sara Kazempour-Ardebili, ${ }^{1}$ Fereidoun Azizi, ${ }^{2}$ Farzad Hadaegh ${ }^{1}$
}

To cite: Mozaffary A, Asgari S, Tohidi M, et al. Change in fasting plasma glucose and incident type 2 diabetes mellitus: results from a prospective cohort study. BMJ Open 2016;6: e010889. doi:10.1136/ bmjopen-2015-010889

- Prepublication history and additional material is available. To view please visit the journal (http://dx.doi.org/ 10.1136/bmjopen-2015010889).

AM and SA contributed equally to this work and are co-first authors.

Received 18 December 2015 Revised 31 March 2016 Accepted 5 April 2016

CrossMark

\begin{abstract}
${ }^{1}$ Prevention of Metabolic Disorders Research Center, Research Institute for Endocrine Sciences (RIES), Shahid Beheshti University of Medical Sciences, Tehran, Islamic Republic of Iran 2Endocrine Research Center, Research Institute for Endocrine Sciences, Shahid Beheshti University of Medical Science, Tehran, Islamic Republic of Iran
\end{abstract}

Correspondence to Farzad Hadaegh; fzhadaegh@endocrine.ac.ir

\section{ABSTRACT}

Objective: To investigate the association between changes in fasting plasma glucose (FPG) values and incident type 2 diabetes (T2D) in a cohort of the Iranian population.

Design: Prospective cohort study.

Setting: This study was conducted within the framework of the Tehran Lipid and Glucose Study (TLGS) to investigate the association between change in FPG between baseline examination (1999-2001) and the second visit (2002-2005) with incident T2D.

Participants: A total of 3981 non-diabetic participants aged $\geq 20$ years.

Outcome measure: T2D was defined if the participant was using antidiabetic drugs or if FPG was $\geq 7 \mathrm{mmol} / \mathrm{L}$ or if the $2 \mathrm{~h}$ post-challenge plasma glucose (2-hPCG) was $\geq 11.1 \mathrm{mmol} / \mathrm{L}$.

Results: During a median follow-up of 6.17 years, after the second examination, 288 new cases of T2D were identified. In a multivariate Cox proportional hazard analysis using age as timescale, we presented a simple model including FPG change (HR $1.19,95 \% \mathrm{Cl}$ 1.07 to 1.33) and baseline waist circumference (WC) (HR $1.004,95 \% \mathrm{Cl} 1.001$ to 1.008 ) with a discriminative power (C-index) of $72 \%$. Furthermore, we showed that the highest quartile of FPG change enhanced the T2D risk to $1.65(95 \% \mathrm{Cl} 1.2$ to 2.27$)$ compared with the lowest quartile ( $p$ for trend $=0.004$ ). The independent risk of FPG change resisted further adjustment with 2-hPCG change. Adding the 2-hPCG change only slightly increased the discriminative power of the model including FPG change and baseline value of WC $(0.73 \%$ vs $0.72 \%)$. After the study population had been limited to those with normal fasting glucose/ normal glucose tolerance, FPG change remained an independent predictor ( $\mathrm{HR} 1.57,95 \% \mathrm{Cl} 1.31$ to 1.88).

Conclusions: Two measurements of FPG obtained about 3 years apart can help to identify populations at risk of incident T2D independently of important traditional risk factors and their changes, including 2-hPCG change.

\section{INTRODUCTION}

Each year over 3.8 million people die from type 2 diabetes (T2D) and its complications

\section{Strengths and limitations of this study}

- The reasonable size of the population.

- Use of direct measurements of the glucose indices and anthropometric data rather than selfreported information for both predictors and outcomes.

- Its design as a long-term community-based prospective study conducted on a large sample of Middle Eastern men and women, a region with high incidence rates of $\mathrm{T} 2 \mathrm{D}$.

worldwide. ${ }^{1}$ The occurrence of T2D has risen rapidly over the past few decades. It has been estimated that between 2010 and 2030, there will be a $69 \%$ increase in numbers of adults with T2D in developing countries and a $20 \%$ increase in developed countries. ${ }^{2}$

Even though most of the investigations have been conducted in Western populations, ${ }^{3-5}$ it seems that the Middle East will have the greatest increase in the prevalence of T2D by 2030. ${ }^{6} 7$ Despite the fact that Middle Eastern populations bear the highest incidence of T2D, data on the dynamics of T2D in this region continue to be lacking.

The rapidly rising prevalence of T2D mandates a more systematic understanding of the natural history of the disease and its potential risk factors. The role of recognised risk factors such as aging, family history of diabetes, obesity, impaired fasting glucose, hypertension, sedentary lifestyle consequent to increasing rates of urbanisation, and some well-known genetic factors have been shown in the occurrence of the disease. ${ }^{7-9}$ However, a complex process exists between these and other risk factors, which varies between populations with different ethnicities and demographic characteristics. ${ }^{10}{ }^{11}$ Investigators have tried to implement risk assessment models that can reflect this complexity and at the same time can be simply used in clinical practice. $^{91213}$ 
Of the different components of T2D risk assessment models, ${ }^{13}$ the level of fasting plasma glucose (FPG) as the core component in most of these models, and its association with increased risk of T2D incidence, has been well investigated. Analysis from the Whitehall II study showed a linear trend of FPG from 13 to 3 years before diagnosis of T2D, which was followed by a quadratic increase starting 3 years before diagnosis of T2D. ${ }^{14}$ Furthermore, the independent association between changes in values of T2D risk factors such as anthropometric measurements ${ }^{15}$ and triglyceride (TG) levels ${ }^{16}$ has been studied. The present study aimed to examine the impact of change in FPG level on T2D prediction during 6 years of follow-up of urban Iranians in a population-based study called the Tehran Lipid and Glucose Study (TLGS).

\section{METHODS}

Study population

The TLGS is a large-scale, longitudinal, communitybased prospective study performed on a representative sample of an urban population of Tehran (the capital of Iran). The study was started in 1999-2001 to estimate the prevalence of non-communicable disease risk factors. Data collection is ongoing and designed to continue for at least 20 years on a triennial basis. The rationale and study design of the TLGS has been discussed elsewhere. ${ }^{17}$

Members of the TLGS who attended both first (19992001) and second (2002-2005) examinations of the cohort were eligible for the present study. Of a total of 10368 individuals aged $\geq 20$ years, after exclusion of participants with prevalent diabetes (having newly diagnosed T2D or using glucose-lowering drugs) ( $\mathrm{n}=1192)$, missing data on FPG and $2 \mathrm{~h}$ post-challenge plasma glucose (2-hPCG) $(\mathrm{n}=4527)$, missing data on other covariates including systolic and diastolic blood pressure (SBP, DBP, respectively), body mass index (BMI), high-density lipoprotein cholesterol (HDL-C), low-density lipoprotein cholesterol (LDL-C), TG, waist circumference (WC), smoking status and education level $(\mathrm{n}=363)$ and no follow-up data $(\mathrm{n}=305), 3981$ subjects were left, who were followed-up until 20 March 2010 (median 6.17 years, IQR 5.4-6.87 years after their second measurement). In the second analysis, we repeated all analyses for participants with normal glucose tolerance (NGT) and normal fasting glucose (NFG) by excluding individuals with FPG $\geq 5.5 \mathrm{mmol} / \mathrm{L}$ and $2-\mathrm{hPCG} \geq 7.7 \mathrm{mmol} / \mathrm{L}$.

The ethics committee of the Research Institute for Endocrine Sciences, Shahid Beheshti University of Medical Sciences approved the design of the TLGS, and all participants provided written informed consent.

\section{Medical history, clinical examination and laboratory measurements}

Participants were interviewed in private by trained interviewers. A standard questionnaire was used to obtain demographic information. Subjects were questioned about their current smoking status and taking of any antidiabetic or hypertension drugs. SBP and DBP were measured twice, with subjects in a seated position, in the right arm using a standard mercury sphygmomanometer; the mean value was considered to evaluate their SBP and DBP.

Weight was measured, with subjects minimally clothed without shoes, using digital scales (Seca 707; range 0.1$150 \mathrm{~kg}$ ) and recorded to the nearest $100 \mathrm{~g}$. Height was measured using a tape measure, with subjects standing without shoes with shoulders in normal alignment. WC was measured at the umbilical level and that of the hip at the maximum level over light clothing, using a tape measure, without any pressure to the body surface; measurements were recorded to the nearest $0.1 \mathrm{~cm}$. BMI was calculated as weight in kilograms divided by height in metres squared.

All measurements of lipid variables and glucose were made in venous samples drawn between 07:00 and 09:00 after 12-14 h overnight fasting into Vacutainer tubes, which were centrifuged within $30-45 \mathrm{~min}$ of collection. FPG and 2-hPCG was measured by an enzymatic colorimetric method using oxidase on the day of blood collection; inter- and intra-assay coefficients of variation $(\mathrm{CVs})$ at baseline and follow-up phases were both $<2.3 \%$.

Total cholesterol (TC) was assayed using the enzymatic colorimetric method with cholesterol esterase and cholesterol oxidase. HDL-C was measured after precipitation of the apolipoprotein B-containing lipoproteins with phosphotungstic acid. TG was assayed using glycerol phosphate oxidase. Both inter- and intra-assay CVs were $<1.9 \%, 3 \%$ and $2.1 \%$ for TC, HDL-C and TG, respectively, in all baseline and follow-up assays. We used a modified Friedewald formula to calculate LDL-C. ${ }^{18}$ Analyses were performed using Pars Azmon kits (Pars Azmon, Tehran, Iran) and a Selectra 2 auto-analyzer (Vital Scientific, Spankeren, Netherlands). All samples were analysed when internal quality control met acceptable criteria.

\section{Definition of terms}

T2D was considered present if the participant was using antidiabetic drugs or if FPG was $\geq 7 \mathrm{mmol} / \mathrm{L}$ or if the 2-hPCG was $\geq 11.1 \mathrm{mmol} / \mathrm{L} .{ }^{19}$ Prediabetes was defined as FPG of $5.6-6.9 \mathrm{mmol} / \mathrm{L}$ and or 2 h-PCG of 7.8 $11.0 \mathrm{mmol} / \mathrm{L} . \quad$ FPG $<5.6 \mathrm{mmol} / \mathrm{L}$ and $2 \mathrm{~h}-\mathrm{PCG}$ $<7.8 \mathrm{mmol} / \mathrm{L}$ was defined as $\mathrm{NFG} / \mathrm{NGT}^{19}$ A current smoker was defined as a person who smokes cigarettes daily or occasionally. A positive family history of T2D was defined as having at least one parent or sibling with T2D. We defined individuals participating in a vigorous physical activity programme of at least 3 days/week as physically active. ${ }^{7}$ We classified amount of education into three groups: <6 years, $6-12$ years and $\geq 12$ years. Hypertension was defined as SBP $\geq 140 \mathrm{~mm} \mathrm{Hg}$ or DBP $\geq 90 \mathrm{~mm} \mathrm{Hg}$ or using any hypertension drugs. 


\section{Statistical analysis}

Mean \pm SD values for continuous variables, and frequencies (\%) for categorical variables, of the baseline characteristics were reported for diabetic and nondiabetic participants. Comparisons of baseline characteristics between respondents (those with FPG and 2-hPCG data in both phases I and II, who had follow-up until the end of the study) and non-respondents (those with missing data on FPG and 2-hPCG and covariates in either phase I or II or loss to follow-up) as well as diabetic versus non-diabetic participants were performed by Student's $\mathrm{t}$ test for continuous variables, the $\chi^{2}$ test for categorical variables, and the Mann-Whitney U statistic for skewed variables.

A univariate analysis was conducted for each potential covariate to be integrated into the multivariate analysis, including gender (female vs male), family history of diabetes, education, current smoking, age, BMI, WC, TG/ HDL-C, TC/HDL-C, LDL-C, physical activity and lipid-lowering drugs as well as change in BMI, WC, TC/ HDL-C, TG/HDL-C, LDL-C, FPG and 2-hPCG and the hypertension change category. Regarding the effect of changes in smoking habits, we created four groups based on the first (1999-2001) and second (2002-2005) examination (yes/yes, yes/no, no/yes and no/no). We also defined the hypertension change category based on the first (1999-2001) and second (2002-2005) examination (yes/yes, yes/no, no/yes and no/no). Variables that had a $\mathrm{p}$ value $<0.2$ in univariate analysis were selected to be included in the multivariate Cox model, with age as the timescale $^{20}$ (see online supplementary table S1).

We checked the interaction between all of the baseline covariates and FPG change in multivariate analysis, and no significant interaction was found. Variance inflation factor was used to check multi-collinearity among anthropometric measurements and their changes (FPG change and 2-hPCG change; lipid measurements and their changes), and there was no multi-collinearity. In the multivariate analysis, three models were designed (the details of covariate adjustment in each model is abbreviated in the tables and defined in footnotes). All adjusted HRs with their 95\% CIs were reported for 1SD increase in each variable and their changing values. In addition, analyses were repeated for quartiles of FPG change.

The proportionality of the multivariate Cox model was evaluated using Schoenfeld's global test of residuals, and all proportionality hypotheses were appropriate. In addition, to better clarify the linear association between FPG change and incident T2D during follow-up, we applied the scaled Schoenfeld proportional hazard assumption graphically. As shown in online supplementary figure S1, no violation of the Schoenfeld proportional hazard assumption was found for FPG changes and incident diabetes during the follow-up.

To measure the relative quality of the predictive models, the Akaike information criterion (AIC) was used. Better fit can be checked with a lower value of
AIC. ${ }^{21}$ Harrell's C-index was used to check the discriminative ability of the models. A value of 1 indicates perfect discrimination, and a value of 0.5 is no better than probability. ${ }^{22}$ All analyses were performed using Stata V.12 SE, with a two-tailed p value $<0.05$ being considered significant.

\section{RESULTS}

Baseline characteristics of subjects with and without incidence diabetes as well as whole population are summarised in table 1. In comparison with individuals without incident T2D, those with T2D were older and less educated. There was no significant difference regarding gender, current smoking status, HDL-C, LDL-C and physical activity levels in subjects with and without incident T2D. The comparison between nonrespondents and respondents is shown in online supplementary table S2. The respondents were older and had higher BMI and DBP, but they had lower rates of smoking and mean level of FPG.

Baseline characteristics of the total study population (3981 cases) in accordance with quartiles of FPG change are shown in online supplementary table S3. As demonstrated, subjects within the highest quartile of FPG change $(\geq 0.4 \mathrm{mmol} / \mathrm{L})$ had higher BMI, TC and TG.

Among the non-diabetic study population, we found 288 (115 men and 173 women) new T2D cases (overall incidence rate 7.84 per 1000 person-years) during a median of 6.17 years of follow-up after second examination measurement.

Results of multivariate Cox proportional hazard analysis for 1SD increase in FPG change and other significant covariates for incidence of T2D among the study populations are given in table 2. Model I showed a positive association between FPG change and T2D incidence. In model II (in the presence of other T2D risk factors as well as their changes), we also found that the FPG change (HR 1.19, 95\% CI 1.07 to 1.33 ) and baseline WC (HR 1.004, 95\% CI 1.001 to 1.008 ) showed positive association with T2D incidence. According to model III, FPG change (HR 1.13, 95\% CI 1.01 to 1.26 ) and 2-hPCG change (HR 1.28, 95\% CI 1.14 to 1.42) remained as independent predictors. We also observed that WC remained in the final model (HR 1.004, 95\% CI 1.001 to 1.008$)$. We did not see any association between other baseline anthropometric and lipid measurements and their changes with T2D incidence (see online supplementary table S4).

With respect to the discriminative power, most differences among the models' C-index were seen between model I and II (model II vs model I: $72 \%$ to $55 \%$ ). As reported by AIC, FPG change had the best fit in model III (AIC 3619) followed by model II and model I.

We also checked the association between the incidence of T2D and quartiles of FPG change. A total of 96 incident cases of T2D were identified in the highest quartile (incident rate 11.63 per 1000 person-year) in 
Table 1 Baseline characteristics of participants with and without incident diabetes and total study population; Tehran Lipid and Glucose Study (TLGS) (2001-2010)

\begin{tabular}{|c|c|c|c|c|}
\hline Characteristic & $\begin{array}{l}\text { Total study } \\
\text { population }(\mathrm{N}=3981)\end{array}$ & $\begin{array}{l}\text { Without incident } \\
\text { diabetes }(\mathrm{N}=3693)\end{array}$ & $\begin{array}{l}\text { With incident } \\
\text { diabetes }(N=288)\end{array}$ & p Value \\
\hline Female & $2297(57.7)$ & $2124(57.5)$ & $173(60.1)$ & 0.4 \\
\hline Age (years) & $42.68 \pm 13.32$ & $42.24 \pm 13.32$ & $48.26 \pm 12.0$ & $<0.001$ \\
\hline Education & & & & $<0.001$ \\
\hline$<6$ years & $1370(34.4)$ & 1231 (33.3) & $139(48.3)$ & \\
\hline $6-12$ years & $2118(53.2)$ & $1994(54.0)$ & $124(43.1)$ & \\
\hline$\geq 12$ years & $439(12.4)$ & $468(12.7)$ & $25(8.7)$ & \\
\hline Family history of diabetes & $1027(25.8)$ & 929 (25.2) & $98(34.0)$ & 0.003 \\
\hline Current smokers & $483(12.1)$ & $457(12.4)$ & $26(9.0)$ & 0.09 \\
\hline Body mass index $\left(\mathrm{kg} / \mathrm{m}^{2}\right)$ & $26.90 \pm 4.45$ & $26.72 \pm 4.38$ & $29.3 \pm 4.66$ & $<0.001$ \\
\hline Systolic blood pressure $(\mathrm{mm} \mathrm{Hg})$ & $118.24 \pm 17.38$ & $117.67 \pm 17.21$ & $125.64 \pm 17.85$ & $<0.001$ \\
\hline Diastolic blood pressure $(\mathrm{mm} \mathrm{Hg})$ & $77.58 \pm 10.23$ & $77.25 \pm 10.14$ & $81.81 \pm 10.45$ & $<0.001$ \\
\hline Total cholesterol (mmol/L) & $5.43 \pm 1.15$ & $5.4 \pm 1.13$ & $5.81 \pm 1.24$ & $<0.001$ \\
\hline High-density lipoprotein cholesterol (mmol/L) & $1.1 \pm 0.28$ & $1.1 \pm 0.28$ & $1.07 \pm 0.27$ & 0.15 \\
\hline Low-density lipoprotein cholesterol (mmol/L) & $3.03 \pm 1.14$ & $3.04 \pm 1.13$ & $2.93 \pm 1.27$ & 0.14 \\
\hline Triglyceride* $(\mathrm{mmol} / \mathrm{L})$ & $1.61(1.22)$ & $1.60(1.19)$ & $2.07(6.53)$ & $<0.001$ \\
\hline Fasting plasma glucose $(\mathrm{mmol} / \mathrm{L})$ & $5.0 \pm 0.51$ & $4.93 \pm 0.50$ & $5.46 \pm 0.58$ & $<0.001$ \\
\hline $2 \mathrm{~h}$ post-challenge plasma glucose ( $\mathrm{mmol} / \mathrm{L})$ & $5.86 \pm 1.55$ & $5.75 \pm 1.48$ & $7.26 \pm 1.75$ & $<0.001$ \\
\hline \multicolumn{5}{|l|}{ Physical activity } \\
\hline More than 3 times a week & $999(25.1)$ & $923(25.0)$ & $76(26.4)$ & 0.6 \\
\hline \multicolumn{5}{|l|}{ Medication use } \\
\hline Hypertension drug & $243(6.1)$ & $210(5.7)$ & $33(11.5)$ & $<0.001$ \\
\hline Lipid drug & $92(2.3)$ & $78(2.1)$ & $14(4.9)$ & 0.003 \\
\hline
\end{tabular}

Values are mean \pm SD (continuous variables) with $p$ value calculated with Student's t test, or $n(\%)$ (categorical variables) with $p$ value calculated according to $\chi^{2}$.

*Triglyceride is shown as median (inter-quartile range) and the $p$ value was calculated according to the Mann-Whitney $U$ test. Low-density lipoprotein cholesterol was calculated using the modified Friedewald formula test.

comparison with the lowest quartile (incident rate 6.85 per 1000 person-year) (table 3). Crude HRs for prediction of T2D across quartiles of FPG change reached 1.71 (95\% CI 1.24 to 2.33) for the highest quartile compared with the lowest $(\mathrm{p}$ for trend $=0.001$ ). With further adjustment in model II, the HR of FPG change decreased to $1.65(95 \%$ CI 1.20 to 2.27$)$ for the highest quartile compared with the lowest quartile ( $p$ for trend $=0.004$ ). After

Table 2 HRs for predicting type 2 diabetes in different models of FPG changes; Tehran Lipid and Glucose Study (TLGS) $(2001-2010)^{*}$

\begin{tabular}{llllll}
\hline & Significant covariatest & HR $(95 \%$ Cl) & p Value & Harrell's C & AIC \\
\hline Model I & FPG change & $1.22(1.09$ to 1.36$)$ & 0.001 & 0.55 & 3701 \\
Model II & FPG change & $1.19(1.07$ to 1.33$)$ & 0.002 & 0.72 & 3637 \\
& WC & $1.004(1.001$ to 1.008$)$ & 0.009 & & 3619 \\
Model III & FPG change & $1.13(1.01$ to 1.26$)$ & 0.03 & \\
& 2-hPCG change & $1.28(1.14$ to 1.42$)$ & $<0.001$ & & \\
& WC & $1.004(1.001$ to 1.008$)$ & 0.012 & & \\
\hline
\end{tabular}

Model I: FPG change (crude model). Model II: model I+baseline measurements of current smoking, family history of diabetes, education, BMI, WC, TG/HDL-C ratio and its change, TC/HDL-C ratio and its change, lipid drug and hypertension change categories. Model III: model II +2 -hPCG change.

We defined the hypertension change category based on the first (1999-2001) and second (2002-2005) examination (yes/yes, yes/no, no/yes and no/no).

*Multivariate Cox analyses were conducted using age as timescale; 1SD change in FPG: $0.5 \mathrm{mmol} / \mathrm{L}$; $1 \mathrm{SD}$ change in 2-hPCG: $1.57 \mathrm{mmol} / \mathrm{L}$; $1 S D$ change in baseline value of WC: $11.5 \mathrm{~cm}$.

tCovariates with $\mathrm{p}$ value $<0.05$ are shown.

2-hPCG, $2 \mathrm{~h}$ post-challenge plasma glucose, mmol/L; AIC, Akaike information criterion; BMI, body mass index; FPG, fasting plasma glucose, $\mathrm{mmol} / \mathrm{L} ; \mathrm{HDL}-\mathrm{C}$, high-density lipoprotein cholesterol; TC, total cholesterol; TG, triglycerides; WC, waist circumference. 
Table 3 HRs $(95 \% \mathrm{Cl})$ for prediction of type 2 diabetes with respect to FPG change quartiles; Tehran Lipid and Glucose Study (TLGS) (2001-2010)

\begin{tabular}{|c|c|c|c|c|c|c|c|}
\hline Quartile of interest & 1 (lowest) & 2 & 3 & 4 (highest) & $\mathbf{p}_{\text {trend }}$ & Harrell's C & AIC \\
\hline FPG change, mmol/L* & $<-0.29$ & $-0.29-0.06$ & $0.07-0.39$ & $\geq 0.40$ & - & - & - \\
\hline Diabetes cases & 64 & 71 & 57 & 96 & - & - & - \\
\hline Person-years & 9335 & 9981 & 9162 & 8253 & - & - & - \\
\hline $\begin{array}{l}\text { Incidence (per } 1000 \\
\text { person-year) }\end{array}$ & 6.85 & 7.11 & 6.22 & 11.63 & - & - & - \\
\hline Model I & 1.00 & $1.08(0.77$ to 1.52$)$ & $0.96(0.67$ to 1.37$)$ & 1.71 (1.24 to 2.33$)$ & 0.001 & 0.65 & 3700.65 \\
\hline Model II & 1.00 & $1.11(0.79$ to 1.56$)$ & $0.98(0.68$ to 1.40$)$ & 1.65 (1.2 to 2.27$)$ & 0.004 & 0.72 & 3636.42 \\
\hline Model III & 1.00 & $1.06(0.76$ to 1.5$)$ & $0.9(0.63$ to 1.29$)$ & $1.43(1.03$ to 1.98$)$ & 0.06 & 0.73 & 3619.2 \\
\hline
\end{tabular}

further adjustment in model III, we found that this risk decreased to 1.43 (95\% CI 1.03 to 1.98 ) (table 3).

Finally, we removed all prediabetes cases at baseline and limited the study population to NGT/NFG participants. All analyses were repeated for these subgroups and results were similar to the main study population. Accordingly, FPG change remained an independent predictor (HR 1.57, 95\% CI 1.31 to 1.88) (see online supplementary tables S5 and S6).

\section{DISCUSSION}

To the best of our knowledge, this study is among the few prospective population-based ones to examine the association of changes in FPG and 2-hPCG with incident T2D. Our results reveal that, after adjustment for traditional risk factors and their changes, baseline $\mathrm{WC}$ and change in FPG and 2-hPCG remained significant predictors. Furthermore, we showed that data on FPG change in combination with a baseline value of WC had a discriminative power of $72 \%$ in the prediction of T2D. However, adding the 2-hPCG change only slightly increased the discriminative power of model II $(0.73 \%$ vs $0.72 \%$ ). We also showed that the change in FPG, even in subjects with NFG and NGT at baseline, was a significant predictor of T2D.

Numerous epidemiological studies have demonstrated that baseline FPG and 2-hPCG are highly predictive of T2D. ${ }^{9} 142324$ Nevertheless, the association between changing the values of different T2D risk factors and incident T2D has been investigated. Tirosh $e t a l^{16}$ in a cohort of young men showed the relation between changes in TG levels and increased risk of T2D. In addition, the association between changes in anthropometric measurements and incident dysglycaemia has been investigated in the TLGS. ${ }^{15}$
Many different risk scores and models have been developed that estimate the risk of T2D, but most are hardly ever used because they involve tests not routinely used or they were developed without a specific user. ${ }^{25} 26$ In a population sample including 3140 men and women in the Framingham Offspring Study, Wilson et $a l^{27}$ showed that, instead of using complex risk models, utilising a combined simple clinical and personal model can efficiently identify subjects at elevated risk of T2D. Applying a simple risk score, we showed that a cluster of data, including SBP, family history of T2D, TG/HDL-C and FPG level, revealed an area under receiver operating characteristic (AROC) of $85 \% .^{9}$

There is now growing evidence that central adiposity correlates better with T2D than general adiposity, which has made WC a more suitable T2D risk predictor than BMI. ${ }^{28} 29$ The finding of this obvious advantage of WC over BMI observed in many studies can probably be explained by the physiological functions of visceral fat tissue, which is known to have endocrine functions and to be an independent risk factor for T2D. ${ }^{28}{ }^{30}$ In this regard, Mamtani et $a l^{31}$ in a study conducted on 808 members of Mexican American families, has shown that $\mathrm{WC}$ is the strongest anthropometric index that is associated with insulin resistance and T2D. Moreover, our results reveal that, after adjustment for traditional risk factors and their changes, WC remained a significant predictor of T2D.

Subjects with impaired glucose tolerance (IGT) are at increased risk of developing T2D and it has become the standard method for recognising people at risk of T2D. ${ }^{32}$ However, detection of IGT requires a costly and time-consuming oral glucose tolerance test (OGTT). Furthermore, it has been shown that only $50 \%$ of IGT subjects converted to T2D within 10 years of follow-up. ${ }^{32}$ Thus, investigators have developed risk assessment models based on fasting state values, which perform 
equally well in predicting future T2D in comparison with the $2 \mathrm{~h}$ plasma glucose concentration during the OGTT. ${ }^{33-38}$ In addition, Stern et $a l^{33}$ showed that adding the 2-hPCG value to the prediction model results in only a slight improvement, at a greater cost and inconvenience. In our previous study, we showed that adding 2-hPCG to the simple model, despite achieving statistical significance, failed to reach clinical importance. ${ }^{9}$ In the present study, we showed that model III, which included 2-hPCG change, outperformed model II with respect to Harrell's C-index and highlighted better fit considering the AIC. However, this gain was clinically negligible. In accordance with Stern et al, ${ }^{33}$ our results show that adding 2-hPCG change to the prediction model did not appreciably increase the predictive power.

In our data analyses we also showed that even those with NFG/NGT at baseline highlighted significant risk of changing values of FPG regarding incident T2D. Recently, a J-shaped FPG-diabetes relationship was shown in the Tehranian adult population. In other words, the risk of T2D increased linearly in individuals with FPG $\geq 5.05 \mathrm{mmol} / \mathrm{L}^{39}$ In fact, applying a simple T2D risk score, we found that $5 \mathrm{mmol} / \mathrm{L} \leq \mathrm{FPG}$ level $\leq 5.5 \mathrm{mmol} / \mathrm{L}$ had a score of 12 in the prediction of T2D. ${ }^{9}$ Hence, it might be predictable that a significant association between change in FPG value and incident T2D in subjects with NFG/NGT had occurred in those with $\mathrm{FPG} \geq 5 \mathrm{mmol} / \mathrm{L}$.

The strengths of the present prospective study are the reasonable size of the population and the use of direct measurements of glucose indices and anthropometric data rather than self-reported information for both predictors and outcomes. A further strength is its design as a long-term community-based prospective study conducted on a large sample of Middle Eastern men and women, a region with high incidence rates of T2D. ${ }^{7}$ Some limitations of the study, which are inherent to any prospective study, need to be addressed. About $57 \%$ of the study participants with missing data or lost to follow-up were not examined at follow-up. However, respondents were older and had a higher BMI, but a lower rate of current smoking and lower level of FPG compared with non-respondents; hence, this might not significantly affect the incidence of T2D in this population. Moreover, our subjects were selected from a sample of the Iranian population, and further studies should be conducted to determine whether our findings are applicable to other populations and ethnicities. Unfortunately, we did not have any data on the changes in physical activity or nutritional habits of the study population during the study; however, true assessments of these measures can be very difficult to achieve with adequate precision. ${ }^{40}$

In summary, we have shown that, after adjustment for traditional risk factors for T2D and their changes, there is a positive association between FPG change and the incidence of T2D. Furthermore, we have also shown that, even in subjects with NFG and NGT, the same positive association exists. We present a simple model, including WC and FPG change with high discriminative power in the prediction of T2D. Our findings also suggest that adding 2-hPCG change to the enhanced prediction model slightly improves the predictive power but at greater cost and inconvenience. Finally, it should be considered that attenuating the change in FPG value through alteration of nutritional habits, physical activity level or pharmacological therapy may potentially weaken the risk of T2D in the Iranian population.

Acknowledgements This study was supported by Grant No 121 from the National Research Council of Iran. The funding source had no role in the design, in the collection, analysis, and interpretation of data, in the writing of the manuscript, and in the decision to submit the manuscript for publication. We express appreciation to the participants of district No13 of Tehran for their enthusiastic support in this study.

Contributors Conception and design, or analysis and interpretation of data, or both: FH, AM, SA. Drafting the article or revising it critically for important intellectual content: FH, AM, SA, MT, SK-A, FA. All authors contributed to the writing of the paper, and read and approved the final manuscript.

Funding This research received no specific grant from any funding agency in the public, commercial or not-for-profit sectors.

\section{Competing interests None declared.}

\section{Patient consent Obtained.}

Ethics approval Ethics committee of the Research Institute for Endocrine Sciences, Shahid Beheshti University of Medical Sciences approved the design of the TLGS, and all participants provided written informed consent.

Provenance and peer review Not commissioned; externally peer reviewed.

Data sharing statement Additional data can be accessed via the Dryad data repository at http://datadryad.org/ with the doi:10.5061/dryad.2517b.

Open Access This is an Open Access article distributed in accordance with the Creative Commons Attribution Non Commercial (CC BY-NC 4.0) license, which permits others to distribute, remix, adapt, build upon this work noncommercially, and license their derivative works on different terms, provided the original work is properly cited and the use is non-commercial. See: http:// creativecommons.org/licenses/by-nc/4.0/

\section{REFERENCES}

1. Yoon U, Kwok LL, Magkidis A. Efficacy of lifestyle interventions in reducing diabetes incidence in patients with impaired glucose tolerance: a systematic review of randomized controlled trials. Metab Clin Exp 2013;62:303-14.

2. Shaw JE, Sicree RA, Zimmet PZ. Global estimates of the prevalence of diabetes for 2010 and 2030. Diabetes Res Clin Pract 2010;87:4-14.

3. Cowie CC, Rust KF, Byrd-Holt DD, et al. Prevalence of diabetes and high risk for diabetes using $\mathrm{A} 1 \mathrm{C}$ criteria in the U.S. population in 1988-2006. Diabetes Care 2010;33:562-8.

4. Soriguer F, Goday A, Bosch-Comas A, et al. Prevalence of diabetes mellitus and impaired glucose regulation in Spain: the Di@ bet.es Study. Diabetologia 2012;55:88-93.

5. Carstensen B, Kristensen JK, Ottosen P, et al. The Danish National Diabetes Register: trends in incidence, prevalence and mortality. Diabetologia 2008;51:2187-96.

6. Wild S, Roglic G, Green A, et al. Global prevalence of diabetes: estimates for the year 2000 and projections for 2030. Diabetes Care 2004;27:1047-53

7. Derakhshan A, Sardarinia M, Khalili D, et al. Sex specific incidence rates of type 2 diabetes and its risk factors over 9 years of follow-up: Tehran Lipid and Glucose Study. PLOS ONE 2014;9:e102563.

8. Hitman GA, Sudagani J. Searching for genes in diabetes and the metabolic syndrome. Int J Clin Pract 2004;58(Suppl 143):3-8.

9. Bozorgmanesh $\mathrm{M}$, Hadaegh $\mathrm{F}$, Ghaffari $\mathrm{S}$, et al. A simple risk score effectively predicted type 2 diabetes in Iranian adult population: population-based cohort study. Eur J Public Health 2011;21:554-9. 
10. Hippisley-Cox J, Coupland C, Robson J, et al. Predicting risk of type 2 diabetes in England and Wales: prospective derivation and validation of QDScore. BMJ 2009;338:b880.

11. Meigs JB, Shrader P, Sullivan LM, et al. Genotype score in addition to common risk factors for prediction of type 2 diabetes. $N$ Engl $J$ Med 2008;359:2208-19.

12. Simmons RK, Unwin N, Griffin SJ. International diabetes federation: an update of the evidence concerning the prevention of type 2 diabetes. Diabetes Res Clin Pract 2010;87:143-9.

13. Noble D, Mathur R, Dent T, et al. Risk models and scores for type 2 diabetes: systematic review. BMJ 2011;343:d7163.

14. Tabák AG, Jokela M, Akbaraly TN, et al. Trajectories of glycaemia, insulin sensitivity, and insulin secretion before diagnosis of type 2 diabetes: an analysis from the Whitehall II study. Lancet 2009;373:2215-21.

15. Hadaegh $F$, Jahangiri Noudeh $\mathrm{Y}$, Tohidi $\mathrm{M}$, et al. Change in general and central adiposity measures in prediction of incident dysglycemia; Tehran Lipid and Glucose Study. Prev Med 2012;55:608-12.

16. Tirosh A, Shai I, Bitzur R, et al. Changes in triglyceride levels over time and risk of type 2 diabetes in young men. Diabetes Care 2008;31:2032-7.

17. Azizi F, Ghanbarian A, Momenan AA, et al. Prevention of non-communicable disease in a population in nutrition transition: Tehran Lipid and Glucose Study phase II. Trials 2009;10:5.

18. Chen $Y$, Zhang $X, P a n B$, et al. A modified formula for calculating low-density lipoprotein cholesterol values. Lipids Health Dis 2010;9:52

19. American Diabetes Association. Standards of medical care in diabetes-2013. Diabetes Care 2013;36(Suppl 1):S11-66.

20. Pencina MJ, Larson MG, D'Agostino RB. Choice of time scale, and its effect on significance of predictors in longitudinal studies. Stat Med 2007;26:1343-59.

21. Agresti A. An introduction to categorical data analysis. Vol 135 New York: Wiley, 1996.

22. Pencina MJ, D'Agostino RB. Overall $\mathrm{C}$ as a measure of discrimination in survival analysis: model specific population value and confidence interval estimation. Stat Med 2004;23:2109-23.

23. Strandberg AY, Pienimäki T, Pitkälä KH, et al. Comparison of norma fasting and one-hour glucose levels as predictors of future diabetes during a 34-year follow-up. Ann Med 2013;45:336-40.

24. Danaei G, Finucane MM, Lu Y, et al. National, regional, and global trends in fasting plasma glucose and diabetes prevalence since 1980: systematic analysis of health examination surveys and epidemiological studies with 370 country-years and 2.7 million participants. Lancet 2011;378:31-40.

25. Wareham NJ, Griffin SJ. Risk scores for predicting type 2 diabetes: comparing axes and spades. Diabetologia 2011;54:994-5.

26. Chen L, Magliano DJ, Balkau B, et al. AUSDRISK: an Australian Type 2 Diabetes Risk Assessment Tool based on demographic, lifestyle and simple anthropometric measures. Med $\mathrm{J}$ Aust 2010;192:197-202.

27. Wilson PW, Meigs JB, Sullivan L, et al. Prediction of incident diabetes mellitus in middle-aged adults: the Framingham Offspring Study. Arch Intern Med 2007;167:1068-74.

28. Hartwig S, Kluttig A, Tiller D, et al. Anthropometric markers and their association with incident type 2 diabetes mellitus: which marker is best for prediction? Pooled analysis of four German population-based cohort studies and comparison with a nationwide cohort study. BMJ Open 2016;6:e009266.

29. Feng R-N, Zhao C, Wang C, et al. BMI is strongly associated with hypertension, and waist circumference is strongly associated with type 2 diabetes and dyslipidemia, in northern Chinese adults. $J$ Epidemiol 2012;22:317-23.

30. Yoon K-H, Lee J-H, Kim J-W, et al. Epidemic obesity and type 2 diabetes in Asia. Lancet 2006;368:1681-8.

31. Mamtani M, Kulkarni H, Dyer TD, et al. Waist circumference independently associates with the risk of insulin resistance and type 2 diabetes in Mexican American families. PLOS ONE 2013;8: e59153.

32. Unwin N, Shaw J, Zimmet $\mathrm{P}$, et al. Impaired glucose tolerance and impaired fasting glycaemia: the current status on definition and intervention. Diabet Med 2002;19:708-23.

33. Stern MP, Williams K, Haffner SM. Identification of persons at high risk for type 2 diabetes mellitus: do we need the oral glucose tolerance test? Ann Intern Med 2002;136:575-81.

34. Aekplakorn W, Bunnag P, Woodward M, et al. A risk score for predicting incident diabetes in the Thai population. Diabetes Care 2006;29:1872-7.

35. Wannamethee SG, Shaper AG, Lennon L, et al. Metabolic syndrome vs Framingham Risk Score for prediction of coronary heart disease, stroke, and type 2 diabetes mellitus. Arch Intern Med 2005;165:2644-50.

36. Kanaya AM, Fyr CLW, de Rekeneire N, et al. Predicting the development of diabetes in older adults: the derivation and validation of a prediction rule. Diabetes Care 2005;28:404-8.

37. McNeely MJ, Boyko EJ, Leonetti DL, et al. Comparison of a clinical model, the oral glucose tolerance test, and fasting glucose for prediction of type 2 diabetes risk in Japanese Americans. Diabetes Care 2003;26:758-63.

38. Lindström J, Tuomilehto J. The diabetes risk score: a practical tool to predict type 2 diabetes risk. Diabetes Care 2003;26:725-31.

39. Bozorgmanesh M, Hadaegh F, Saadat N, et al. Fasting glucose cutoff point: where does the risk terminate? Tehran lipid and glucose study. Acta Diabetol 2012;49:341-8.

40. Sullivan LM, Massaro JM, D'Agostino RB. Presentation of multivariate data for clinical use: the Framingham Study risk score functions. Stat Med 2004;23:1631-60. 